\title{
Consenso sobre mobiliario infantil seguro. Versión abreviada
}

\author{
Consensus on safe infant's furniture. Brief version
}

Comité Nacional de Prevención de Lesiones

Véase el texto completo en formato electrónico, en la sección "Consensos" del sitio web de la Sociedad Argentina de Pediatría www.sap.org.ar

\section{RESUMEN}

Diversosproductosqueseutilizanhabitualmente para el sostén, el transporte o el entretenimiento de niños pequeños pueden causar lesiones no intencionales. El presente consenso intenta brindar a pediatras y familias elementos para conocer y elegir el mobiliario infantil seguro. El consenso convocó a 24 expertos y se realizó siguiendo el método Delphi, que consiste en rondas sucesivas de preguntas. Las recomendaciones están avaladas con bibliografía sobre cada tema.

Se recomienda no utilizar andadores, por ser inútiles y peligrosos.

Se dan pautas para elegir un sistema de retención infantil adecuado a cada niño, cómo y cuándo utilizarlo y cómo instalarlo en forma segura. Se describen las lesiones y sus medidas de prevención en relación con cochecitos para niños, sillas altas, cunas y cuchetas.

Se esquematizan los riesgos y las formas de evitarlos mediante figuras, que podrían utilizarse para difundir recomendaciones en las familias.

Palabras clave: seguridad, mobiliario infantil, andadores, sistemas de retención infantil, cuchetas.

\begin{abstract}
Several products that are used for support, transportation or recreation in infants and children can cause non intentional injuries. This consensus tries to provide pediatricians and families with the necessary elements to recognize and choose safe infant's furniture.

A group of 24 experts developed a consensus according to Delphi's method, which consists in successiveroundsofquestions. Recommendations are supported with bibliography.

Infant walkers are not recommended, as they are considered useless and dangerous.

Guidelines are given to choose appropriate child restraint systems, when and how to use them, and how to install them in a safe way.

Injuries and prevention measures related to strollers, high chairs, cribs and bunk beds are described.

Risks and the way to avoid them are diagrammed in figures that can be used to transmit recommendations to families.

Key words: safety, infant furniture, infant walkers, child restraint systems, bunk beds.
\end{abstract}

http:/ /dx.doi.org/10.5546/aap.2016.182
Correspondencia:

Dra. Ingrid Waisman, cnplsap@sap.org.ar; ingridwaisman@arnet. com.ar

Financiamiento: Ninguno.

Conflicto de intereses: Ninguno que declarar.

Recibido: 28-10-2015 Aceptado: 5-11-2015

\section{Coordinadores}

Dra. Ingrid Waisman, Dr. Rubén Zabala, Dra. Clotilde Ubeda.

\section{Participantes del consenso}

Adela Armando, Osvaldo Aymo, Mariela Bustamante, Natalia Caorsi, Juan Dartiguelongue, Guillermo Dehoyos, Sandra Ferraro, Romina Ferreyra, Jorge Fiorentino, Ana Garbocci, Cristina Gatica, María Eugenia Gordillo, Alejandro Jenik, Verónica Llorens, Benjamín Malamud, Liliana Méndez Castell, Lucas Navarro, Marcelo Orlievski, Andrea Perinetti, Valeria Rodríguez Alcántara, Norma Rossato, Gustavo Sastre, Sergio Snieg, Patricia Stach, Eduardo Zori.

\section{Integrantes del Comité Nacional de Prevención de Lesiones}

Daniel Miranda, Ingrid Waisman, Clotilde Ubeda, Rubén Zabala, Osvaldo Aymo, Andrea Perinetti, Paulina Cendrero, Cristina Gatica, Carlos Nasta, Lucas Navarro, Adela Armando, Luis Agote, Lorenzo Sambuelli. 


\section{INTRODUCCIÓN}

Diversos productos que se utilizan habitualmente para el sostén, el transporte o el entretenimiento de niños pequeños pueden causar lesiones no intencionales. En algunos países, la fabricación y la venta de dichos productos están legisladas y reglamentadas, y es obligatorio que los productos que se fabrican y venden se encuentren homologados. ${ }^{1,2}$

En Argentina, no hay leyes que regulen la calidad y seguridad de los productos destinados a los niños. Creemos que es una deuda pendiente brindar asesoramiento a las familias para que puedan conocer y elegir el mobiliario que se considera adecuado y seguro.

Los objetivos del consenso son los siguientes:

- Capacitar a los pediatras para asesorar a los padres sobre las características que debe tener el mobiliario infantil y su utilización segura.

- Elaborar material que facilite tal asesoramiento en la consulta y/o salas de espera.

- Intervenir en medios de comunicación masivos para asesorar a la comunidad.

- Solicitar al organismo estatal competente la elaboración de normas de calidad y seguridad.

Para desarrollar este consenso, se convocó a un panel de 24 expertos y se utilizó una adaptación del método Delphi. ${ }^{3,4}$ Este consiste en realizar rondas sucesivas de preguntas sobre cada tema e identificar coincidencias y desacuerdos. Se repreguntó a los expertos sobre los desacuerdos para intentar consensuar recomendaciones generales. Las conclusiones tienen, además, una sólida base bibliográfica.

\section{ANDADORES}

Los padres colocan a sus hijos en andadores para entretenerlos, para mantenerlos ocupados, porque piensan que es un lugar seguro, porque creen que los ayuda a caminar con mayor rapidez y facilidad.

Sin embargo, los niños que utilizan andador retrasan su maduración para la deambulación, tienen dificultades para desarrollar el equilibrio, no gatean lo suficiente y caminan más tarde. ${ }^{(5,6)}$

Los andadores son responsables de diversas lesiones. La mayoría de las lesiones graves se deben a caídas por las escaleras. Otros mecanismos de lesión son vuelcos o desplomes, atrapamiento de dedos y acceso a diversos peligros. Las heridas incluyen traumatismos craneoencefálicos, fracturas, quemaduras, lesiones dentales, laceraciones e intoxicaciones. ${ }^{7,8}$
A pesar de ser usados bajo la supervisión de un adulto, la velocidad que pueden alcanzar los niños en los andadores es mayor que aquella a la que puede reaccionar el adulto, por lo que quedan expuestos a velocidades, distancias o alturas que no pueden manejar.

Pese a que la mayoría de los pediatras desaconseja su utilización, el porcentaje de uso de andadores en Argentina va desde el 40\% hasta el $72 \%$. Muchos niños que utilizaron andadores sufrieron lesiones que requirieron consultas, y un $47 \%$ de los niños que consultaron a la Guardia por lesiones relacionadas con andadores requirió internación. ${ }^{9}$

La mayoría de los trabajos publicados coinciden en desaconsejar su uso por la ausencia de beneficios y el riesgo de lesiones. Canadá fue, en 2004, el primer país del mundo que prohibió la publicidad, venta e importación de andadores.

En los Estados Unidos, se fijaron normas de seguridad en la fabricación de andadores, que lograron una marcada disminución en el número de lesiones. ${ }^{8}$

\section{Medidas de prevención}

El rol del pediatra es importante pero no suficiente para desalentar el uso de andadores. Durante la consulta, muchas veces, se pierden oportunidades de prevención por diversas razones. ${ }^{10}$ La acción aislada del pediatra no alcanza y debemos bregar por intervenciones más efectivas.

La Sociedad Argentina de Pediatría desaconseja el uso de andadores. Como una primera etapa, se recomienda fomentar campañas educativas acerca del riesgo, para luego intentar una legislación adecuada.

\section{SISTEMAS DE RETENCIÓN INFANTIL}

Las lesiones por transporte son una de las primeras causas de muerte y secuelas graves en niños y jóvenes. Para evitar las consecuencias de un choque, existen dispositivos de seguridad pasiva, que constituyen medidas efectivas de prevención secundaria. ${ }^{11}$

\section{Cinturones de seguridad y sistemas de retención infantil}

El lazo superior del cinturón debe pasar por el tórax y la zona clavicular, y la parte inferior, por la raíz de los muslos; la columna del niño debe apoyar sobre el respaldo. Estas condiciones se logran entre los 8 y los 12 años de edad y a los $150 \mathrm{~cm}$ de altura. ${ }^{12}$ 
Un niño transportado en los brazos de un adulto, al sufrir una colisión, multiplica su peso y resulta imposible sostenerlo. Esto le ocasionará serias lesiones al ser despedido o al ser golpeado dentro del vehículo. ${ }^{11}$

Existen importantes diferencias entre bebes y niños respecto a los adultos. Es por ello por lo que se utilizan sistemas de retención que se adaptan a sus características anatómicas. Los sistemas de retención infantil (SRI) reducen las defunciones de lactantes un $71 \%$ y las de niños pequeños un $54 \%{ }^{13}$

El niño debe ubicarse sentado mirando hacia atrás para evitar la hiperflexión cervical en caso de choque frontal durante el mayor tiempo posible.

Pese a que los SRI constituyen probadas medidas de seguridad, su uso no está difundido y solo es obligatorio por ley en algunas ciudades. Según estimaciones de diversos organismos, solo una minoría de pasajeros los utiliza. ${ }^{14,15}$

\section{Legislación}

En Argentina, existe una heterogeneidad inadmisible en cuanto a la utilización de medidas de seguridad y, en particular, al uso de SRI. ${ }^{16}$ La legislación es desigual, incompleta y desactualizada.

Por encima de los mínimos legislativos, es importante que los pediatras aconsejemos a los padres lo que tiene probado beneficio en la prevención de las lesiones de transporte.

\section{Tipos de sistemas de retención infantil}

Las sillas se clasifican según el peso y la talla del niño en grupos: $0,0+, 1,2$ y 3 . Sus características e indicaciones pueden observarse en la Figura 1.

El SRI se debe elegir según la edad y el peso del niño, así como por la facilidad de instalación en el automóvil. Se aconseja leer el manual del vehículo, las instrucciones del fabricante del SRI y llevar al niño y el coche en el momento de comprar la sillita.

Se recomienda elegir productos certificados; no utilizar sillas usadas que hayan sufridos golpes o con piezas faltantes y sustituirlas después de 6 años de uso.

\section{Instalación del sistema de retención infantil}

La fijación deberá ser adecuada. En caso de una colisión, no sirve de nada tener una buena silla mal colocada. ${ }^{11}$

Actualmente, coexisten tres sistemas de anclajes, que ofrecen buenas condiciones de seguridad: el sistema tradicional, que utiliza los cinturones del vehículo; el sistema ISOFIX, con ganchos fijos; y el sistema LATCH, con ganchos y correas.

El SRI irá colocado en la plaza central del asiento trasero del automóvil, pues es la que ofrece mayor seguridad en caso de colisión.

\section{Recomendaciones ${ }^{11,17}$}

- Todos los niños deben viajar en un SRI mirando hacia atrás hasta los 2 años o hasta alcanzar el peso y la talla máximos permitidos.

- En viajes largos, cuando es necesario alimentar o atender a niños pequeños, se sugiere detener el vehículo para hacerlo.

- Los niños que han superado el límite de peso y de altura máximo del SRI anterior pueden utilizar un SRI mirando hacia adelante. Luego de superar los límites de este SRI, utilizarán dispositivos elevadores.

- Aquellos mayores de 12 años o de 1,50 m utilizarán el cinturón de seguridad provisto por el automóvil.

- Todos los menores de 12 años deben viajar en el asiento trasero.

- El niño debe viajar dentro de un SRI desde la salida de la maternidad. Aún en trayectos cortos, todos los niños deben utilizar las sillitas para viajar seguros.

- Las sillitas se utilizan exclusivamente para el transporte de los niños. Se desaconseja el uso del "huevito" como lugar de juego o descanso.

- Transporte en motocicletas: no existen sistemas de protección para niños. La recomendación es NO transportar a niños menores de 12 años en estos vehículos.

\section{COCHECITOS PARA BEBES}

Un cochecito es un vehículo con ruedas para el transporte de bebes o niños. Hay recomendaciones aplicables a todos (calidad de los materiales, estado de conservación, ruedas grandes y con sistema de frenado, sujeción con sistemas de retención infantil), pero otras que son específicas para cada modelo. Es recomendable leer los manuales de instrucción para asegurar un armado y uso seguro.

Se describen como lesiones más frecuentes las caídas; además, la estrangulación, la asfixia, los traumatismos múltiples (especialmente, de cráneo), las lesiones o las amputaciones parciales o totales de dedos, la incapacidad ventilatoria, las lesiones en la piel, el aplastamiento, las quemaduras. ${ }^{18,19}$ 


\section{Tipos de cochecitos}

Cochecito clásico o coche cuna: el bebe está recostado frente a la persona que lo empuja. Tiene la forma de una pequeña cuna y se asienta sobre un chasis con ruedas. Llevan a los niños en él desde que nacen hasta alrededor de 3 o 4 años.

Coche paragüita: la principal característica es su liviandad y su plegado compacto y sencillo.

Sistemas polivalentes: consisten en un chasis, un asiento y una cuna de bebe desmontable, que se encajan en el chasis según la edad del niño.

Sistemas 3 en 1: permiten, además de un asiento y una cuna desmontable, encajar sobre el chasis la silla de bebe para el automóvil.

Coche de tres ruedas: posee 3 grandes ruedas ( 2 traseras, 1 delantera) y los frenos se accionan desde el mango. Se adapta a superficies irregulares.

En la Figura 2, se resumen los riesgos de los distintos componentes del cochecito y las medidas de prevención.

\section{Precauciones $^{20,21}$}

Al colocar al niño

- Controle la posición, con la cabeza extendida, con adecuado ajuste de cinturones.

- Las manos y los pies, libres al abrocharle el arnés.

- En la posición reclinada, cierre las aberturas de las piernas para que no pueda deslizarse.

- Sujete las mantas; no deje elementos sueltos.

\section{Al circular con el coche}

- Utilice siempre los arneses de seguridad.

- El niño debe estar siempre bajo supervisión directa.

- Debe circular con un adulto que no tenga limitaciones en su movilidad.

- Circule exclusivamente por la vereda; cruce por la senda peatonal; respete las reglas de tránsito. Espere en la vereda para cruzar.

- No desatienda al niño durante el traslado.

- No lo use en escaleras mecánicas.

\section{Al dejar el coche con el niño adentro}

- Asegure el frenado de las ruedas; no lo deje expuesto al sol; no sobreabrigue al niño.

- No deje colgantes que puedan favorecer la autoestrangulación.

- La vigilancia debe ser continua.

\section{Al retirarlo}

- Siempre por un adulto, con el coche frenado; no realice movimientos bruscos y retírelo acompañando la cabeza y el tronco.

- Libere la fijación; controle que los miembros no queden atrapados.

- En un niño que deambula, vigile que, al bajar, no corra hacia la calle.

\section{SILLAS ALTAS O TRONAS}

Son sillas donde se sienta a un niño a la altura de una mesa de comedor y se utilizan para la alimentación. Pueden contar con bandejas fijas o removibles. Pueden tener ruedas para favorecer su traslado y ser plegables para facilitar su almacenamiento.

Las sillas altas pueden causar lesiones en los niños, por fallas en el producto y/o prácticas inseguras en su uso. ${ }^{22,23}$ Son más frecuentes las lesiones por caídas y menos frecuentes las quemaduras y otras lesiones. La sujeción correcta del niño evita la mayoría de las lesiones. .2,24 $^{2}$

La Figura 3 resume los riesgos y las recomendaciones para el uso de sillitas.

\section{Recomendaciones ${ }^{1,25}$}

Al elegir una silla alta

- Es conveniente que no sea muy alta.

- Utilice sillas sin ruedas.

- Con base de sustentación amplia para minimizar el riesgo de vuelco.

- Asegure la integridad de las patas de la silla, así como de los forros, para evitar que quede expuesta la gomaespuma y permitir una buena higiene.

- Coloque gomas en las patas de las sillitas.

- Sistema de sujeción: cinturón de 5 puntos o, al menos, de 3 puntos. Con hebillas seguras, fáciles de operar para los adultos y a prueba de niños.

- Bandejas con mecanismos seguros de fijación, que impidan su desprendimiento o el atrapamiento de dedos.

- Material: sin aristas, bordes afilados, materiales tóxicos o piezas pequeñas que puedan desprenderse.

- Mantenga la silla en buen estado de conservación e higiene.

- En sillas usadas: observe que sean de buena calidad, no deterioradas.

- No use las antiguas sillas de madera. No compre sillas en lugares inseguros: internet o usadas.

Al usar la silla alta

- El niño debe encontrarse sujeto siempre y con supervisión permanente. 
- Úsela solo en el momento de la comida y con un adulto que acompañe.

- No permita ni favorezca movimientos o juegos bruscos.

- No deje que los niños mayores se suban o cuelguen de la silla.

- Advierta el riesgo de que el niño puede impulsarse con sus pies y volcar la silla.

- Si la silla tiene ruedas, bloquéelas.

Al ubicar la silla alta

- Apóyela sobre una superficie firme, con un centro de gravedad adecuado.

- No la ubique en una superficie mojada o resbaladiza.

- Manténgala lejos de ventanas, electrodomésticos, correas de persianas, enchufes, tóxicos y medicamentos y todo otro elemento peligroso.

- Lejos de objetos en los que pueda apoyarse y empujar.

- Aleje objetos atractivos del campo visual del niño.

- Quite el mantel de la mesa.

- Luego de utilizarla, pliéguela o guárdela.

\section{CUNAS SEGURAS}

La cuna es un tipo de cama para niños pequeños con barrotes laterales para evitar caídas. Son fabricadas con diferentes materiales, como madera, metal o plástico.

Se utilizan desde los 2 o 3 meses hasta que el niño alcanza $90 \mathrm{~cm}$ de altura.

Los lactantes pasan gran parte de su tiempo en la cuna, que es considerada un espacio seguro; sin embargo, las cunas, a veces, presentan riesgos. ${ }^{26}$ Las lesiones pueden ir desde caídas, intoxicaciones y quemaduras hasta muertes por asfixia o síndrome de muerte súbita de la infancia. ${ }^{23,27}$

Los riesgos pueden ser generados por mal diseño, fabricación defectuosa, armado equivocado o uso inadecuado. ${ }^{26}$

La Figura 4 resume las recomendaciones de una cuna segura y su uso adecuado.

\section{Recomendaciones}

\section{Al elegir la cuna}

Materiales: madera, metal o plástico sin astillas, tornillos expuestos, puntas agudas o aristas filosas. Los ángulos deben ser redondeados. La pintura, sin sustancias tóxicas. ${ }^{25}$

Barrotes laterales: el espacio entre barrotes debe ser menor de $6 \mathrm{~cm}$ y mayor de $4,5 \mathrm{~cm}$.
La altura del lateral debe ser, por lo menos, de $60 \mathrm{~cm}$. Evite la colocación de juguetes o mantas.

No se recomiendan las cunas con barandas móviles. ${ }^{25,28,29}$

Estabilidad: son más seguras las cunas con patas firmes y fijas. En caso de tener ruedas, se recomienda un mecanismo seguro de bloqueo. Los pernos y tornillos deben estar bien ajustados. Se recomienda probar la estabilidad de la cuna. Si se tambalea o hace ruidos, es probable que el armado sea incorrecto. ${ }^{28}$

Esquineros: se deben evitar los esquineros grandes o con perillas decorativas que puedan enganchar la ropa o collares del niño.

Evite los espacios de atrapamiento. ${ }^{29}$

Cunas usadas: los riesgos por fragilidad, inestabilidad, partes faltantes o rotas y errores de reensamblado aumentan con los años y el uso. Las cunas antiguas son atractivas por su apariencia, pero muy inseguras en su diseño.

\section{Colchones}

El colchón debe ser de material firme. Vigile roturas para evitar que el relleno de gomaespuma se escape. . $5,30^{25}$

Debe ser del tamaño exacto de la cuna. No debe haber más de $2 \mathrm{~cm}$ entre el borde del colchón y la cuna. ${ }^{25,30}$

\section{Uso seguro de la cuna}

Evite elementos colgantes (sogas, hilos, juguetes colgantes, móviles, etc.), pues pueden provocar autoestrangulamiento. No deje, en el entorno, fundas plásticas ni objetos pequeños.

La cuna debe colocarse, al menos, a $90 \mathrm{~cm}$ de enchufes, cables y correas.

Ropa blanda: las almohadas, edredones, acolchados y otras superficies suaves son peligrosos en el entorno del sueño; pueden aumentar el riesgo de muerte inesperada del lactante y asfixia accidental. Por los riesgos de sofocación, no se recomiendan los protectores de cunas (chichoneras) ni tampoco los posicionadores o nidos. ${ }^{31}$

Sueño seguro durante el primer año de vida $a^{31,32}$

Recomendamos que el bebe duerma boca arriba, en un colchón firme de igual tamaño que la cuna, sin objetos dentro de ella, tapado hasta las axilas con los brazos por fuera.

Se recomienda que comparta la habitación de los padres, pero no la cama.

Evite el exceso de abrigo y la temperatura ambiente elevada. No exponga al niño al 
humo del tabaco desde el embarazo. Se debe alimentarlo con leche materna y ofrecer el chupete para dormir cuando la lactancia esté bien establecida.

Son riesgosos para dormir, en los primeros meses de vida, los siguientes lugares: la cama de los padres, los "huevitos" (solo deben utilizarse para el transporte), las eslingas y los columpios.

\section{CAMAS CUCHETAS, LITERAS O MARINERAS}

La cama cucheta está formada por dos o más camas superpuestas. El soporte donde asienta el colchón de la cama superior se encuentra a más de $81 \mathrm{~cm}$ del piso. Su uso es muy frecuente para ahorrar espacio en viviendas pequeñas en familias con más de un hijo.

Los lesionados más frecuentes son los varones $y$, a pesar de que no debe ser utilizada por niños menores de 6 años, la mitad de los afectados son de esa edad. ${ }^{33}$

El mecanismo de lesión más común asociado a cuchetas son las caídas, pero también pueden producirse estrangulación o ahorcamiento accidental, atrapamiento de extremidades, caída de la cama superior sobre la inferior, lesiones por ventiladores de techo o lámparas.

Las lesiones mayores son politraumatismos, fracturas de cráneo, contusiones cerebrales, fracturas de huesos largos y lesiones de bazo. Las lesiones menores incluyen fracturas menores, contusiones y torceduras, laceraciones de piel y fracturas dentales. ${ }^{33,34}$

\section{Recomendaciones}

La Figura 5 resume los riesgos y las recomendaciones en el uso de cuchetas.

No se recomienda el uso de camas marineras para niños en general y, en especial, el uso de la parte superior de la cama cucheta para menores de 6 años. Si, por motivos de espacio, tiene que utilizarla, le recomendamos lo siguiente:

\section{Elija el lugar correcto ${ }^{35}$}

- Coloque la cama en un rincón.

- No coloque la cama cerca de un ventilador de techo.

- Coloque una luz nocturna cerca de la escalera.

- Mantenga despejada y ordenada el área del piso circundante.

\section{Elija cuchetas seguras ${ }^{36}$}

- Si compra un mueble para armar, siga las instrucciones de montaje con cuidado.
- Revise la estructura para detectar posibles grietas, tornillos sueltos o puntos débiles.

- Asegúrese de que el soporte del lecho superior sea fuerte.

- Asegúrese de que la escalera no esté suelta o rota.

- Ponga barandas a ambos lados de la litera de arriba.

- No deben quedar espacios entre la baranda y el colchón.

- Utilice los colchones de tamaño correcto.

- Homologación y certificación del mueble.

3. Eduque a los niños para el uso correcto de las cuchetas ${ }^{35}$

- Hable con sus hijos sobre la seguridad en la litera.

- Los niños menores de 6 años no deben dormir ni jugar en la litera de arriba.

- Muéstreles cómo utilizar la escalera.

- La cucheta no debe ser utilizada como un juego.

- No permita que haya más de un niño en la cama superior.

- Nunca cuelgue o ate pañuelos, cuerdas, cinturones o bufandas de la litera de arriba.

\section{REFERENCIAS}

1. Sengölge M, Vincenten J. Child Product Safety Guide: potentially dangerous products. Birmingham: European Child Safety Alliance, EuroSafe; 2013. [Acceso: 5 de noviembre de 2015]. Disponible en: http://www. childsafetyeurope.org/publications/info/product-safetyguide.pdf.

2. Hagan JF, Shaw JS, Duncan PM. Bright futures. Guidelines for Health Supervision of Infants, Children, and Adolescents. $3^{\text {rd }}$ ed. Elk Grove Village: American Academy of Pediatrics; 2008.

3. García Valdés M, Suárez Marín M. El método Delphi para la consulta a expertos en la investigación científica. Rev Cuba Salud Pública 2013;39(2):253-67.

4. Rivara FP, Johansen JM, Thompson DC. Research on injury prevention: topics for systematic review. Inj Prev 2002;8(2):161-5.

5. Siegel AC, Burton RV. Effects of baby walkers on motor and mental development in human infants. J Dev Behav Pediatr 1999;20(5):355-61.

6. Garrett M, McElroy AM, Staines A. Locomotor milestones and babywalkers: cross sectional study. BMJ 2002;324(7352):1494.

7. Esparza Olcina MJ. Prevención de lesiones infantiles por accidente doméstico [Internet]. Madrid: Recomendaciones Prev Infad/PAPPS; 2011. [Acceso: 5 de noviembre de 2015]. Disponible en: http:/ / www.aepap.org/previnfad/ accidentes_domesticos.htm.

8. Shields BJ, Smith GA. Success in the prevention of infant walker-related injuries: an analysis of national data, 19902001. Pediatrics 2006;117(3):e452-9.

9. Waisman I, Ubeda C, Zabala R. Lesiones por andadores registradas en Sistema de Vigilancia de Lesiones (SI.VI.LE.) [abstract]. En:Sociedad Argentina de Pediatría. $6{ }^{\circ}$ Congreso 
Argentino de Pediatría General Ambulatoria;2014 Nov 1921; Buenos Aires, Argentina.

10. Waisman I. Prevención de lesiones de 0 a 3 años. PRONAP 2013. Módulo $\mathrm{N}^{\circ}$ 2. Buenos Aires: SAP; 2013.Págs.9-30.

11. Navarro LD. ¿Qué deben saber los pediatras sobre las sillitas para autos? Sistemas de retención infantil para automóviles. Arch Argent Pediatr 2013;111(3):247-50.

12. FiorentinoJA, Dell'olio A. Seguridad dentro del automóvil y los niños pasajeros. Rev Hosp Niños B Aires 2013;55(249): 97-107.

13. US Department of Transportation. National Highway Traffic Safety Administration. Report to Congress: Child Restraint Systems, Transportation Recall Enhancement, Accountability, and Documentation Act, February 2004. [Acceso: 5 de noviembre de 2015]. Disponible en: http:// www.nhtsa.gov/Research/Child+Seat+Research.

14. Argentina. Ministerio del Interior y Transporte. Dirección Nacional de Observatorio Vial [Internet]. [Acceso: 5 de noviembre de2015]. Disponibleen:http:/ /observatoriovial. seguridadvial.gov.ar.

15. Luchemos por la Vida. Estudio sobre niños dentro del automóvil [Internet]. Buenos Aires: Luchemos por la Vida; 2009. [Acceso: 5 de noviembre de 2015]. Disponible en: http://www.luchemos.org.ar/es/investigaciones / estudio-sobre-ninos-dentro-del-automovil.

16. Fiorentino J. Aspectos relacionados con el transporte vehicular seguro. Rev Hosp Niños B Aires 2013;55(249):84-5.

17. Asociación Española de Pediatría. Viajes seguros: sistemas de retención infantil. [Acceso: 5 de noviembre de 2015]. Disponible en: http://enfamilia.aeped.es/prevencion/ nino-viaja-seguro-sistemas-retencion-infantil.

18. Chowdhury RT. Injuries and deaths associated with nursery products among children younger than age five. Bethesda: Consumer Product Safety Commission; 2012 [Acceso: 5 de noviembre de2015]. Disponibleen: http:/ / www.cpsc.gov / PageFiles/136143/nursery11.pdf.

19. Powell EC, Jovtis E, Tanz RR. Incidence and description of stroller-related injuriestochildren.Pediatrics 2002;110(5):e62.

20. Watson WL, Ozanne-Smith J. The use of child safety restraints with nursery furniture. J Paediatr Child Health 1993;29(3):228-32.

21. US Consumer Product Safety Commission. Safety Alert: Infants in strollers must be properly secured at all times serious risk of entrapment and strangulation. Bethesda: CPSC; 2010. [Acceso: 5 de noviembre de 2015]. Disponible en: http:/ / www.cpsc.gov//PageFiles/113400/5096.pdf.

22. Kurinsky RM, Rochette LM, Smith GA. Pediatric injuries associated with high chairs and chairs in the United States, 2003-2010. Clin Pediatr (Phila) 2014;53(4):372-9.

23. MackKA, Gilchrist J, Ballesteros MF. Injuries among infants treated in emergency departments in the United States, 2001-2004. Pediatrics 2008;121(5):930-7.
24. Powell EC, Jovtis E, Tanz RR. Incidence and description of high chair-related injuries to children. Ambul Pediatr 2002;2(4):276-8.

25. Subcomisión de Prevención de Lesiones. Manual de prevención de lesiones. Buenos Aires: Sociedad Argentina de Pediatría; 2012.

26. Yeh ES, Rochette LM, McKenzie LB, Smith GA. Injuries associated with cribs, playpens, and bassinets among young children in the US, 1990-2008. Pediatrics 2011;127(3):479-86.

27. Shapiro-Mendoza CK, Kimball M, Tomashek KM, Anderson RN, et al. US infant mortality trends attributable to accidental suffocation and strangulation in bed from 1984 through 2004: are rates increasing? Pediatrics 2009;123(2):533-9.

28. Comisión de Seguridad de Productos del Consumidor de EE. UU. Para que su bebe duerma seguro, primera parte: la cuna. [Acceso: 5 de noviembre de 2015]. Disponible en: http://onsafety.cpsc.gov/es/2010/02/18/para-que-subebe-duerma-seguro-primera-parte-la-cuna/.

29. European Child Safety Alliance. Consumer rights for child safety products. Final report. Amsterdam: European Child Safety Alliance; 2004. [Acceso: 5 de noviembre de 2015]. Disponible en: http://unsafeproducts.eu/tips / 25dangers-to-children/.

30. Liga de Acción del Consumidor. Cunas: ¿están seguros los bebes? [Acceso: 5 de noviembre de 2015]. Disponible en: http://www.adelco.org/images/productos/Cunas_ Seguras.pdfbebe

31. Fundación para el Estudio y la Prevención de la Muerte Infantil y Perinatal. Ropa de cama y aumento del riesgo del Síndrome de Muerte Súbita del Lactante. [Acceso: 5 de noviembre de 2015]. Disponible en: http:/ / www.sids.org. ar/bolsadedormir1.html.

32. Grupo de Trabajo en Muerte Súbita e Inesperada del Lactante. Consideraciones sobre el sueño seguro del lactante. Resumen ejecutivo. Arch Argent Pediatr 2015;113(3):285-7.

33. Mack KA, Gilchrist J, Ballesteros MF. Bunk bed related injuries sustained by young children treated in emergency departments in the United States, 2001-2004, National Electronic Injury Surveillance System - All Injury Program. Inj Prev 2007;13(2):137-40.

34. Gelfman MG, Ledesma J, Huier F, Volonté P, et al. Trauma por caída de altura en pediatría. Arch Argent Pediatr 2005;103(5):414-9.

35. Rasch $\mathrm{H}$. Rest easy with these bunk bed safety tips. AAP News 2012;33(7)

36. US Consumer Product Safety Commission. Bunk Beds Business Guidance. [Acceso: 5 de noviembre de 2015]. Disponible en: http://www.cpsc.gov/en/Business-Manufacturing/Business-Education/Business-Guidance/ Bunk-Beds/. 